\title{
Semiotik
}

Band $37 \cdot$ Heft 1-2 (2015)

Seite 23-53

Stauffenburg Verlag Tübingen

\section{Psychologische Ansätze zur Erforschung des Lachens}

\author{
Sabine Kowal, Berlin \\ Daniel C. O'Connell, Washington DC
}

\begin{abstract}
Seit mehr als zweitausend Jahren haben sich Gelehrte intensiv mit dem Wesen und der Bedeutung von Humor und Lachen beschäftigt und eine beachtliche Anzahl von Meinungen zu diesem Thema vorgebracht. Zurückblickend können wir jedoch nicht behaupten, dabei große Fortschritte erzielt zu haben; das Thema ist rätselhaft geblieben (Milner 1972: 1). ${ }^{1}$
\end{abstract}

Summary. The following article provides a selective review of more than 130 years of research on the definition, types, and functions of adult vocal laughter from the point of view of psychology. The review shows that laughter has not been extensively researched, although it has been claimed to be a promising topic for psychologists. Empirical approaches have, in the course of time, focused increasingly on the communicative functions of laughter in spoken dialogue. Methodologically, this approach has included the interdisciplinary efforts of phonetics, linguistics, and conversation analysis. The field-observational research of the present authors, presented in summary fashion, has conceptualized laughter as a paralinguistic, nuanced rhetorical tool in the hands of experts (Hannah Arendt) and politicians (Hillary and Bill Clinton) in media interviews, and actors in the movie The Third Man and in the BBC mini-series Pride and Prejudice. The findings indicate that the two types of laughter (HA-HA laughter and suprasegmental laughter) may have different communicative functions, that gender may be an important variable in determining type, amount, duration, and function of laughter, that vocal laughter is not necessarily related to humorous content, and that HA-HA laughter may be functionally similar to interjections.

Zusammenfassung. Der folgende Aufsatz gibt eine selektive Zusammenfassung der mehr als 130-jährigen Geschichte und des gegenwärtigen Forschungsstandes zu Definition, Arten und Funktionen des vokalen Lachens von Erwachsenen aus psychologi- 
scher Perspektive. Der Überblick zeigt, dass das Lachen bisher zwar nur sporadisch untersucht wurde, zugleich aber als vielversprechendes Thema betrachtet wird. Im Laufe der Zeit ist in empirischen Untersuchungen die kommunikative Funktion des Lachens in gesprochenem Dialog in den Mittelpunkt gerückt. Methodologisch ist dieser Forschungsansatz interdisziplinär mit Phonetik, Linguistik und Konversationsanalyse verknüpft. In unserer eigenen Feldforschung haben wir das Lachen speziell als ein rhetorisches Mittel konzeptualisiert, das von ExpertInnen (Hannah Arendt) und Politikerlnnen (Hillary und Bill Clinton) in Medieninterviews sowie von Schauspielerlnnen in dem Film The Third Man und in der BBC Mini-Serie Pride and Prejudice verwendet wird, um individuelle Einstellungen mit Hilfe von paralinguistischen Mitteln indirekt auszudrücken. Die Ergebnisse weisen darauf hin, dass HA-HA-Lachen und suprasegmentales Lachen unterschiedliche kommunikative Funktionen haben können, dass das soziale Geschlecht wesentlich die Art, die Häufigkeit und die Funktion des Lachens bestimmen kann, dass Lachen nicht notwendig im Kontext humorvoller Äußerungen auftritt und dass HA-HALachen funktionale Ähnlichkeiten mit Interjektionen aufweist.

\section{Einleitung}

Der folgende Aufsatz beschäftigt sich mit dem Phänomen des Lachens bei Erwachsenen aus psychologischer Perspektive, genauer gesagt mit dem Lachen als vokalem Verhalten in der dialogischen Interaktion von Erwachsenen. Nicht berücksichtigt werden Beschreibungen des Lachens in schriftlichen Texten (z.B. Sidis 1913), visuell wahrnehmbare Aspekte des Lachens (z.B. Ruch und Ekman 2001) und Lächelns (z.B. Fridlund 1991; Jaffe 2010) sowie körperliche Begleiterscheinungen des Lachens (z.B. Romaniuk 2013). Methodologisch erfordert der hier vertretene empirische Ansatz eine Reihe von Entscheidungen: über die Methoden der Datenerhebung (Experiment vs. Feldbeobachtung), über reliable und valide Methoden der Identifizierung des Lachens, über operationale Definitionen der verschiedenen Arten und Dimensionen des Lachverhaltens, über den Einsatz von Transkripten, die dem Forschungsziel angemessen sind (z.B. O'Connell und Kowal 2009), über instrumentelle Methoden zur Messung von vokalen Aspekten des Lachens und über den Einsatz von quantitativen und qualitativen Methoden. Aufgrund der thematischen Schwerpunktsetzung ergeben sich Überschneidungen zur Phonetik, Linguistik und zur Konversationsanalyse. In unseren eigenen empirischen Untersuchungen des Lachens stehen dessen sozialpsychologische Funktionen im Mittelpunkt. Aus diesem Grund gehen wir auf evolutionsbiologisch orientierte Ansätze, die sich mit der phylo- und ontogenetischen Entwicklung des Lachens beschäftigen, nur am Rande ein.

Oberflächlich betrachtet scheint das Lachen als vokales Verhalten ein eindeutig bestimmbares, unkontroverses psychologisches Thema zu sein: Menschen lachen, wenn sie etwas komisch oder humorvoll finden. Tritt ein solches Verhalten in einer sozialen Situation auf, ist es häufig ansteckend. 
Die Beispiele in Abschnitt 2 zeigen jedoch, dass das Lachen tatsächlich ein komplexes soziales Phänomen ist. Auf der produktiven Seite ist zu berücksichtigen, dass es nicht immer etwas Komisches ist, was uns zum Lachen bringt (Abschnitt 2.1). Auf der rezeptiven Seite ist zu berücksichtigen, dass das Lachen neben dem positiven Ansteckungseffekt auch negative Reaktionen auslösen kann (Abschnitt 2.2). Und die in Abschnitt 2.3 aufgeführten Studien aus evolutionsbiologischer Perspektive zeigen die Vielfalt der funktionalen Interpretation des Lachens. Der in Abschnitt 3 dargestellte historische Forschungsüberblick weist auf die anhaltende Suche nach einer adäquaten Definition sowie nach möglichen Funktionen und Ursachen des Lachens in der psychologischen Forschung hin. Die Abschnitte 4 und 5 geben Einblicke in grundlegende methodologische Details psychologischer Forschungsarbeiten zum Lachen, und in Abschnitt 6 fassen wir einige Ergebnisse unserer eigenen Feldstudien zum Lachen in dialogischer Interaktion von Erwachsenen zusammen. In Abschnitt 7 wird die geringe Beschäftigung mit dem Lachen in der psychologischen Forschung zum Teil darauf zurückgeführt, dass traditionell deren grundlegende Analyseeinheit das Individuum ist, während das Lachen wesentlich an soziale, dialogische Interaktion gebunden ist, das heißt an Analyseeinheiten, die mindestens zwei wechselseitig aufeinander bezogene Personen umfassen.

\section{Das Lachen - ein „chamäleonartiges“ Verhalten (Black 1984: 2995)}

\subsection{Lachen vs. Humor}

Interessanterweise bezieht sich ein großer Teil der Studien zum Lachen nicht auf das Lachverhalten selbst, sondern auf die Phänomenbereiche Witz, Humor, Komödie und das Groteske. In der Philosophie und Literatur ging es zumeist um die Frage, was uns zum Lachen bringt, statt zu fragen, wie wir lachen:

In drei Jahrhunderten Neuzeit haben ganze Kohorten von Philosophen, Ästhetikern, Theologen, Literaten, Weltleuten über das Lächerliche und das Komische nachgedacht. Nur das Lachen kam kaum in den Blick - er blieb fixiert auf seine Auslöser. (Seibt 2002: 760)

Bücher über das Lachen drücken diesen Widerspruch bereits in ihren Titeln aus, so zum Beispiel Bergsons (1900/1914) Le Rire: Essai sur la Signification du Comique. Apte (1985) hat Armstrong (1928), Grotjahn (1957), Hertzler (1970) und Piddington (1933) dafür getadelt, „die Begriffe ,Lachen“ und ,Humor" synonym“ (274) verwendet zu haben; ein aktuelleres Beispiel ist das Buch Laughing Matters: A Serious Look at Humour (1988) von Durant und Miller. Ebenso problematisch ist Martins (2001) Subsumierung des Lachens unter den Begriff Humor: „der Begriff Humorkann verwendet werden, um sich auf einen Auslöser (z.B. einen Komödienfilm), auf einen men- 
talen Prozess (z.B. die Wahrnehmung oder Herstellung belustigender Inkongruenzen) oder auf eine Reaktion (z.B. Lachen, Heiterkeit) zu beziehen“ (505). Provine (2000) kommt in seinem historischen Überblick von „philosophischen und theoretischen Ansätzen des Lachens“ (11) zu dem Schluss, dass diese sich kaum jemals mit dem Lachen selbst beschäftigt haben und dass die „laughterless study of laughter“ (18) bis in die Gegenwart reicht.

Erst im zwanzigsten Jahrhundert standen die akustischen, respiratorischen und elektronischen Instrumente bereit, die für die Identifikation, Messung, Transkription und Verhaltensanalyse des Lachens gebraucht wurden. Und erst allmählich wurden Veröffentlichungen, die sich mit Humor und dem Komischen beschäftigen - neben den von Apte (1985) erwähnten könnte man z.B. noch Gregory (1924) und Greig (1923/1969) nennen von linguistischen, konversationsanalytischen und psychologischen Publikationen abgelöst, die sich tatsächlich mit dem Lachverhalten beschäftigen (z.B. Bachorowski, Smoski und Owren 2001; Glenn 2003; Glenn und Holt 2013; Partington 2006; Provine 2000, 2014). Vor diesem Hintergrund müssen sowohl Chafes (2007) The Importance of not Being Earnest: The Feeling Behind Laughter and Humorals auch Martins (2006) The Psychology of Humor: An Integrative Approach als ein Rückfall in die Fokussierung auf das Thema Humor angesehen werden, obwohl sich beide auch mit dem Lachen auseinandersetzen.

\subsection{Soziale Einstellungen zum Lachen}

Vor mehr als einem Jahrhundert schrieb Sully (1902/2012) zur Einführung in seinen Essay on Laughter.

Ein Autor, der über das Lachen schreibt, stößt auf eine Vielzahl verwirrender Hindernisse. Er muss zu seinem Erschrecken feststellen, dass ein erheblicher Teil seiner Spezies, die schmeichelhaft als lachendes Tier beschrieben worden ist, diese vornehme und einzigartige Fähigkeit niemals praktiziert hat. Nein, mehr noch lernt er bald, dass viele sich dieser Praxis verweigern und das Lachen hassen. Ein solcher Mensch [...] ist so besessen vom Geist der Ernsthaftigkeit, dass ihm das gegenteilige, heitere Naturell als völlig falsch erscheint. Alles hörbare Lachen ist für inn eine unangemessene Zurschaustellung, als körperliche Verrenkung unangenehm zu betrachten und als Entgleisung ernsthafter Vernunft eine Art mentaler Degeneration (Sully 1902/2012: 1).

Der Psychologe Sully hatte, wie man aus seinem Buch erfährt, bei dieser Aussage zwar eher die Einstellung von Philosophen (z.B. Schopenhauer) zum Lachen als die von PolitikerInnen im Sinn. Aber er hätte seine kritische Anmerkung vermutlich in der Forderung des türkischen Vizepremierministers Bülent Arinc bestätigt gesehen, Frauen das herzhafte Lachen in der Öffentlichkeit zu verbieten. Seibert hat dieses Problem am 29. Juli 2014 im Berliner Tagesspiege/ wie folgt kommentiert: 
Das herzhafte Lachen einer Frau in der Öffentlichkeit ist in der Türkei ab sofort eine politische Aktion [...] Arinc hatte einen allgemeinen Verfall der Sitten in der Türkei beklagt und hinzugefügt, die Sittsamkeit sei ein hohes Gut, das gepflegt werden müsse. Bei Frauen gehört laut Arinc dazu, sich zurückhaltend zu kleiden und ,nicht vor allen Leuten laut loszulachen'.

In Reaktion auf die Aussage von Arinc konstatierte Seibert, dass das Lachen von Frauen von nun an als Ausdruck der Opposition gegen eine Regierung angesehen werden kann, die in die Privatsphäre ihrer Bürgerlnnen eingreift.

Eine andere Art herzhaften Lachens von Seiten einer Frau wurde vor vielen Jahren zum politischen Ereignis und erschien 50 Jahre später auf den Kulturseiten deutscher Tageszeitungen (z.B. Krause 2006: 14. Oktober 2006 in welt.de). Anlass war der 100. Geburtstag der politischen Philosophin Hannah Arendt am 10. Oktober 2006. Ihr Lachen wurde von ihr selbst im Verlauf ihres legendären TV-Interviews Zur Person mit Interviewer Günter Gaus aus dem Jahr 1964 thematisiert (vgl. O'Connell und Kowal 1998 in Abschnitt 6.1 des vorliegenden Artikels). Als Arendt von Gaus zu ihrem Buch über den Eichmann-Prozess (Arendt 1964) befragt wurde, das gerade erschienen war und eine überwältigende Polemik auslöste, berichtete sie, wie die an Eichmann zu beobachtende Banalität des Bösen sie zum Lachen brachte:

Sehen Sie, es gibt Leute, die nehmen mir eine Sache übel, und das kann ich gewissermaßen verstehen: Nämlich, daß ich da noch lachen kann. Aber ich war wirklich der Meinung, daß der Eichmann ein Hanswurst ist, und ich sage Ihnen: Ich habe sein Polizeiverhör, 3600 Seiten, gelesen und sehr genau gelesen, und ich weiß nicht, wie oft ich gelacht habe; aber laut! Diese Reaktion nehmen mir die Leute übel. Dagegen kann ich nichts machen (Gaus 1964: 26).

Diese Beispiele zeigen, dass das Lachen, oft nicht mehr als ein freudiges, harmloses Verhalten, das das Wohlbefinden der beteiligten Personen ausdrückt, unter bestimmten Umständen als Verletzung öffentlicher Verhaltensnormen gewertet und deshalb abgelehnt werden kann. Gleichzeitig kann es diese vermeintliche Verletzung des öffentlichen Anstands dem Lachenden selbst, im vorliegenden Fall Hannah Arendt, ermöglichen, „,sich selbst“ vom Massenmörder Adolf Eichmann „zu distanzieren“ (Berliner Zeitung, 27. September 2006: 19). Diese evaluative und funktionale Komplexität wurde bisher in der Geschichte der psychologischen Forschung über das Lachen kaum berücksichtigt.

\subsection{Lachen aus evolutionsbiologischer Perspektive}

In der Science Times-Rubrik der New York Times vom 13. März 2007 (Seiten D1 und D6) erläuterte John Tierney einige neuere Publikationen zum 
Lachen. Typischerweise beziehen sich Science Times-Artikel nur auf wenige Literaturangaben (in diesem Fall Provine 2000 und Panksepp 2005) und zusätzlich auf Interviews mit Personen, die als Experten auf dem Gebiet vorgestellt werden. Derartige Artikel sollen einer breiteren Öffentlichkeit Forschungsergebnisse präsentieren und können deshalb leicht zu stark verallgemeinerten Aussagen führen: „Lachen scheint eher eine automatische Antwort auf eine Situation als eine bewusste Strategie zu sein" (D6) und „Es ist ein instinktives Hilfsmittel des Überlebens für soziale Tiere, nicht eine intellektuelle Antwort auf Witz" (D1). In beiden Zitaten - das erste von einem Interviewten, dem Neuropsychologen Robert R. Provine, das zweite von John Tierney selbst - treten Gegenüberstellungen auf, die wir aus unserer sozialpsychologischen Sichtweise auf das Lachen nicht als Alternativen betrachten. Und beide Paare von Alternativen (automatisch vs. bewusst und instinktiv vs. intellektuell) bleiben unbestimmt in Bezug auf Annahmen über die psychologischen Bedingungen, die das Lachen bei Erwachsenen auslösen können.

Die andere von Tierney verwendete Quelle bezieht sich auf Forschungsarbeiten des Neuropsychologen Jaak Panksepp (2005), der im Schlusssatz seines Aufsatzes warnend feststellte: „Obwohl Einige das Lachen immer noch als eine im Pleistozän entwickelte spezifisch menschliche Fähigkeit betrachten, wird sich ihre Annahme langfristig als falsch erweisen“ (63). Panksepp und Burgdorf (2003) stellten eine Verbindung her zwischen ihren Beobachtungen an Ratten, „primitivem menschlichen Lachen“ und dem „Lachen von Kindern“ (533). Und Panksepp (2007) kam aufgrund einschlägiger Untersuchungen zu dem Schluss, dass mittlerweile genügend formale Ähnlichkeiten zwischen einem Ultraschallzirpen von $50 \mathrm{kZh}$ bei Ratten und menschlichem Lachen gefunden wurden, um begründet anzunehmen, dass die beiden Lautäußerungen homolog sind.

\section{Psychologische Lachforschung im historischen Überblick}

Bereits im neunzehnten Jahrhundert gab es empirische Studien zum Lachen. Zu den frühesten gehört Ewald Heckers (1873) Die Physiologie und Psychologie des Lachens und des Komischen: Ein Beitrag zur experimentellen Psychologie für Naturforscher, Philosophen und gebildete Laien. Der Psychiater Hecker war der Ansicht, dass die enge Verbindung von Physiologie und Psychologie bei der Erzeugung des Lachens es inm gestattete, das Lachen "nach der naturwissenschaftlichen und experimentellen Methode“ (vi) zu untersuchen. Er zitierte Wilhelm Wundts (1862) Beiträge zur Theorie der Sinneswahrnehmungen und Wundts Experimente als Modell für seine eigene Forschung. Für Hecker gehört vokales Lachen, genau wie Niesen und Gähnen, physiologisch zu den „Reflexkrämpfe[n]“(4) und kann durch Kitzeln ausgelöst werden. In seinen auf physiologische Aspekte des Lachens bezogenen Experimenten bat Hecker seine Probanden einen bestimmten Punkt zu fixieren, während sie mit einer Feder gekitzelt wur- 
den. Dieses Vorgehen führte zu einer deutlich wahrnehmbaren Erweiterung ihrer Pupillen. Als psychologisches Äquivalent des Lachens identifizierte Hecker das Komische. Aufgrund eines Literaturüberblicks und seiner eigenen Überlegungen kam er zu dem Schluss, dass die Natur des Komischen psychologisch durch einen „beschleunigten Wettstreit der Gefühle, d.h. als ein schnelles Hin- und Herschwanken zwischen Lust und Unlust" bzw. als "eine intermittierende freudige Erregung“ (81; kursiv für Sperrdruck im Original) begriffen werden kann. Er argumentierte, dass diese freudige Erregung von einer „intermittierende[n] Sympathikusreizung“ (83; kursiv für Sperrdruck im Original) begleitet wird, die er als die physiologische Basis in seinen Kitzel-Experimenten identifiziert hatte. Er war davon überzeugt, dass er auf diese Weise die Psychologie des Komischen in Übereinstimmung mit der Physiologie des Lachens gebracht hatte. Sully (1902/2012) äußerte sich in einer Fußnote kritisch zu Heckers Idee, das Lachen über komische Dinge als „grundsätzlich vergleichbar mit dem Lachen beim Kitzeln“ zu betrachten und es deshalb zur „Grundlage für eine merkwürdige und suggestive Theorie des Lachens" (184) zu machen. Nichtsdestotrotz benutzte der Psychologe Alan J. Fridlund (Fridlund und Loftis 1990) fast 90 Jahre später Fragebögen von Collegestudenten anstelle von Beobachtungen des Lachverhaltens, um „die Verbindungen zwischen Kitzeln und humorvollem Lachen" (Fridlund und Loftis 1990: 141) weiter zu verfolgen. Die Autoren fanden eine „,vorläufige Bestätigung für die Darwin-Hecker-Vermutung, dass diejenigen Reflexe, die der Kitzligkeit zugrunde liegen, Humor auslösen“ (141), ein Ergebnis das Harris und Christenfeld (1997) mit experimentellen Ergebnissen widerlegten (vgl. Provine 2000: 124f.).

Stanley G. Hall und Arthur Allin (1897) sahen - wenngleich mit einer veränderten Methodologie - ebenfalls eine Verbindung zwischen dem Lachen, dem Kitzeln und dem Komischen, zogen jedoch vorsichtigere Schlüsse aus ihren Untersuchungsergebnissen als Hecker. Sie verschickten einen Fragebogen an eine große Anzahl von Probanden, die über ihre Erfahrungen mit diesen Phänomenen berichten sollten. Die Autoren betonten jedoch, dass ihre Resultate als vorläufig zu betrachten seien und kamen zu dem Schluss:

Wir sind davon überzeugt, dass alle gegenwärtigen Theorien völlig unangemessen und spekulativ sind, dass es zugleich jedoch kaum ein vielversprechenderes Thema für die psychologische Forschung gibt. Als nächstes gilt es, alle verfügbaren Möglichkeiten der photographischen Momentaufnahme anzuwenden, um das Lachen und Lächeln von Männern und Frauen, von Kindern und Erwachsenen in allen Phasen ihres Auftretens zu dokumentieren [...] Zweitens sollten die Möglichkeiten der Phonographie für vokale Äußerungen des Lachens genutzt werden. [...] Wir müssen uns von Spekulationen lösen und unsere Theorien auf eine sehr breite empirische Basis stellen (Hall und Allin 1897: 40f.).

Hall und Allin kritisierten ältere theoretische Ansätze zum Lachen als „entweder schlichtweg misslungen und irreführend oder den Feinheiten der 
Natur des Lachens völlig unangemessen oder als bloß literarische Beschreibungen von Teilaspekten des Phänomens“ (Hall und Allin 1897: 41). Erwähnenswert ist, dass sich spätere Lachforscher auf Beispiele aus Hall und Allins Fragebogenauswertungen gestützt haben, um ihre eigenen Lachtheorien empirisch zu untermauern (z.B. Gregory 1924).

Von den an der Wende des neunzehnten zum zwanzigsten Jahrhundert verfügbaren psychologischen Veröffentlichungen über das Lachen ist Sullys (1902/2012) Essay on Laughter: Its Forms, its Causes, its Development and its Value bei weitem die informativste. James Sully (1842-1923), ein „Zu seiner Zeit einflussreicher britischer Psychologe“ (Wertheimer 1979: 82) schrieb ein Buch, das glücklicherweise für wichtig genug gehalten wurde, um in der Classic Reprint Series of Forgotten Books nachgedruckt zu werden. Bereits kurz nach Erscheinen des Buches urteilte Allin (1903: 307), dass Sullys Buch, trotz gewisser Mängel, „bei weitem das beste ist, was bisher [zu diesem Thema] veröffentlicht wurde“. Erwähnenswert ist das Buch wegen seiner umfassenden Darstellung und kritischen Diskussion damaliger philosophischer und sozialwissenschaftlicher Theorien des Lachens, wegen seiner psychologischen Einsichten und nicht zuletzt wegen seiner stilistischen Brillanz.

Sully war besonders gut in der Auswahl plausibler und überzeugender Beispielen, ohne selbst empirische Untersuchungen durchzuführen. Ein Beispiel dafür ist seine Beobachtung, dass das Lachen als Folge einer „frohen Stimmung“ (1902/2012: 72) dadurch verhindert werden kann, dass ein Mensch dieser Stimmung nicht seine volle Aufmerksamkeit schenkt. Sully hob hervor, dass er sich für "das schlichte Lachen von Sterblichen“ (5) interessierte und war deshalb besonders kritisch gegenüber solchen (vor allem philosophischen) Theorien, die sich mit dem Lachen nicht aus dieser Perspektive beschäftigten. Er betonte auch die „weite Bandbreite der Erfahrung, die unser Lachen widerspiegelt“ (17f.). Gleichzeitig bestand er darauf, dass „menschliches Lachen trotz seiner Variabilität, seiner Willkür, Gesetzen gehorcht" (20) - eine Überzeugung, die die Grundannahme der gesprächsanalytischen Forschung um 80 Jahre vorwegnimmt, nach der „ein Interaktionsphänomen wie das Lachen nicht zufällig auftritt, sondern zur Herstellung und Aufrechterhaltung eines bedeutungsvollen Systems beiträgt" (Adelswärd und Öberg 1998: 414). Schließlich betonte Sully bereits am Anfang seines Buches nachdrücklich, er werde versuchen, „die Fehler derer zu vermeiden, die in ihren subtilen Abhandlungen über das Komische vergaßen, dass das Lachen ein körperlicher Akt ist“ (Sully 1902/2012: 22).

Ohne Bezug auf Sully formulierte Sidis (1913) in seiner Psychology of Laughter „das Gesetz, dass alle ungehemmten spontanen Aktivitäten mit normaler Funktion freudige Emotionen auslösen, die im Lächeln und Lachen ihren Ausdruck finden" (3). Aber trotz der impliziten Betonung des Lachens als eines expressiven Verhaltens in seinem Gesetz stellte er fest: „Ich musste meine Beispiele des Komischen aus der Literatur verschiedener Nationen und Epochen wählen“ (ix). Damit vollzog Sidis einen impliziten Schwenk vom vokalen Lachverhalten zum geschriebenen Humor. 
Bliss (1915) kann als Vertreterin einer frühen Entlastungstheorie des Lachens bezeichnet werden. Sie stellte die grundlegende Frage: „Warum lachen wir überhaupt?“ (236) und war überzeugt, dass diese Frage nicht von Philosophen sondern nur von Biologen und Psychologen empirisch zu beantworten sei. Ihre Theorie - explizit auf „das reine, elementare Lachen“ (242) beschränkt - bestand aus der einfachen Annahme, dass das Lachen Symbol für die Befriedigung unbewusster Neigungen ist. Entsprechend behauptete Bliss, „je höher hinauf wir uns in der Skala von Witz und Humor bewegen, desto weniger heftig und ungestüm ist das Lachen“ (243). Und sie fügte hinzu, es sei behauptet worden, dass Amerikaner inzwischen nur noch mental lachten, aus ihrer Sicht ein Hinweis auf die „Fortschritte in der Evolution - ein mentaler Ersatz für primitivere Bedürfnisse und Neigungen“ (243). Mit einem ähnlichen Gewicht auf „unbewussten Neigungen“ betrachtete Carpenter (1922) das Lachen als

den Jubel der Seele über ihre eigene geistige Gesundheit. Oder etwas sachlicher und genauer formuliert, das Lachen drückt eine Emotion aus, die auf die plötzliche Überwältigung des Bewusstseins durch das unbewusst ständig vorhandene Vergnügen an der eigenen Urteilskraft zurückgeht. Diese Emotion wird durch die Überflutung mit Ideen ausgelöst, die erfolglos versuchen die Urteilskraft in die Irre zu führen (Carpenter 1922: 419).

Insgesamt ist festzuhalten, dass der Beginn des zwanzigsten Jahrhunderts nicht gerade eine Oase ernst zu nehmender empirischer Forschung war. Dennoch beobachtete Diserens (1926) bereits wenig später in seiner informativen Zusammenfassung derzeitig aktueller Lachforschung „die Tendenz von intellektuellen Beschreibungen wegzukommen und eine zunehmende Bereitschaft, Phänomene des Lachens in behavioristischen Begriffen zu diskutieren“ (247). In seinem Schlusskommentar notierte er, das Lachen sei eine Aktivität „von amöbenartiger Gestalt“:

Es handelt sich um eine komplexe Art des Verhaltens, die nicht gelernt ist, jedoch leicht in Gegenwart von psychischen Stimuli konditioniert werden kann. Das Lachen ist zugleich ein biologischer Anpassungsmechanismus, ein physiologisches Sicherheitsventil, in psychologischer Hinsicht ein Ausdruck von Erheiterung und zudem ein Regulativ für soziale Beziehungen (Diserens 1926: 254).

Sein Kommentar nimmt erkennbar die Betonung interdisziplinärer Ansätze in der gegenwärtigen Lachforschung vorweg. Vier Jahre später beobachteten Diserens und Bonifield (1930) in einem weiteren Forschungsüberblick ,eine zunehmende Tendenz, soziale Faktoren in der Genese und Funktion des Lachens zu betonen,“ zugleich aber auch, „,dass nur sehr wenige wichtige Beiträge geleistet wurden" (108).

Ein gutes Beispiel für die Betonung der sozialen Funktion des Lachens ist in Hayworth (1928) zu finden. Er drückte sein Missfallen an bestehenden Theorien über Ursprung und Funktion des Lachens aus und schlug 
vor, das Lachen als „Mittel der Kommunikation“ (368) zu konzeptualisieren, das heißt

als ein vokales Signal an Mitglieder der eigenen Gruppe, dass sie sich gefahrlos entspannen können. Sie [die Theorie] geht davon aus, dass das Lachen sich, lange bevor Sprache entstand, als Nebenprodukt schweren Atmens beim Kämpfen oder angehaltenen Atmens bei Anspannung entwickelte. Um seine Wirkung als Signal zu erhöhen, wurden die Lippen zurückgezogen. Auf diese Weise wurde das sichtbare Signal des Lächelns schließlich als Alternative zum hörbaren Lachen verwendet. Als sich im Laufe der Zeit soziale Interaktion entwickelte, war es nur natürlich, dass sich Formen und Funktionen des Lächelns und Lachens verfeinerten (Hayworth 1928: 384).

Im Hinblick auf unseren eigenen Forschungsansatz (vgl. Abschnitt 6) ist seine Einbeziehung der folgenden speziellen Funktionen des Lachens bemerkenswert:

Schließlich gibt es eine Art des Lachens, die sich nicht aus der Situation heraus entwickelt, sondern absichtlich erzeugt wird. [...] Ein boshafter Junge kann seine Lehrerin ärgern, indem er über sie lacht und damit andeutet, dass sie inn nicht beherrschen kann... Lachen kann verwendet werden, um Missachtung mitzuteilen, um Schüchternheit oder Verlegenheit zu überspielen oder um die eigenen Gedanken zu verbergen. In allen diesen Fällen wird das Lachen immer noch für kommunikative Zwecke verwendet, aber es wird absichtlich und nicht spontan, das heißt aus der Situation heraus, erzeugt (Hayworth 1928: 380).

Piddington (1933) betonte in seinem Buch Psychology of Laughter: A Study in Social Adaptation, dass - und dies wird den Leser kaum überraschen keine der existierenden Theorien des Lachens eine „vollständige und angemessene Lösung für das Problem des Lachens anbietet“ (146). Zugleich betonte er, dass das Lachen über etwas Komisches nur durch das Vorliegen sozialer Bedürfnisse erklärt werden kann. Im Anhang seines Buches gab er einen historischen Überblick über Theorien des Lachens, der von Plato bis zu dem Werk des englischen Entwicklungspsychologen C. W. Kimmins (1928) The Springs of Laughter reicht.

Aus sozialpsychologischer Sicht hat Morrison (1940) das Publikumslachen in 13 Aufführungen desselben Theaterstücks empirisch untersucht. Er definierte Lachen „als jede direkt beobachtbare Kehlkopfreaktion des Publikums, die durch das Theaterstück ausgelöst wurde, mindestens eine Sekunde anhielt und auditiv wahrgenommen werden konnte" (180). Morrison fand heraus, dass Häufigkeit und Dauer des Lachens von Aufführung zu Aufführung stark variierten, dass jedoch beide Variablen positiv mit der Größe des Publikums korrelierten: Je größer das Publikum, desto häufiger und anhaltender das Lachen. Er schloss daraus, dass dieses Ergebnis „Allports [1924] Konzept der Sozialen Erleichterung (Social Facilitation) bestätigt“ (185). 
Soziale Aspekte des Lachens standen auch viele Jahre später im Mittelpunkt der Experimente von Chapman (1973a). Ihn interessierte der Effekt von witzigen Tonbandaufnahmen, die mit oder ohne Hintergrundlachen dargeboten wurden, auf beobachtbare heitere Reaktionen der Probanden. Sogenannte „Heiterkeits-Werte“, die auf der Basis von Beobachtungen des Lachens und einem vergnügten Gesichtsausdruck gewonnen wurden, waren in Anwesenheit von Hintergrundlachen signifikant höher als ohne dieses Lachen. Die Ergebnisse wurden später von Martin und Gray (1996) an einer Studie mit britischen StudentInnen bestätigt, die einzeln ein kurzes humorvolles Rundfunkprogramm mit oder ohne natürliches Publikumsgelächter hörten. Das Lachen der Probanden wurde operational in Übereinstimmung mit Chapman (1973a) definiert als „ein unartikuliertes vokales Geräusch hinreichender Intensität, das wahrgenommen werden kann, wenn eine Aufzeichnung davon [...] mit maximaler Lautstärke wiedergegeben wurde" (530). Chapman und Chapman (1974) bestätigten ihre früheren Untersuchungsergebnisse anhand eines weiteren Experiments über soziale Aspekte des Lachens mit sieben- und achtjährigen Kindern: Die Kinder lachten mehr, wenn ihre gleichaltrigen Mitschülerlnnen häufiger lachten.

Duncan und Fiske (1977) analysierten ein umfangreiches Korpus von Face-to-Face-Interaktionen zwischen gleich- und gegengeschlechtlichen Paaren. Die Paare wurden instruiert „sich für etwa sechs oder sieben Minuten miteinander zu unterhalten“ (36). Die Autoren kodierten die letzten Minuten jeder Interaktion im Hinblick auf eine Vielzahl von Verhaltensweisen. Bezüglich des Lachens fanden sie heraus, dass dies in ihrem Korpus „ein ziemlich seltenes Ereignis“ (80) war, aber typischerweise in Reaktion auf das Lachen des Partners oder der Partnerin auftrat. Zusätzlich stellten sie fest, dass Frauen häufiger lachten als Männer.

Adams und Kirkevold (1978) ließen in inrer Feldstudie in drei Restaurants jeweils für drei Minuten Gäste beim Abendessen beobachten. Die geschulten Beobachterlnnen notierten verschiedene Verhaltensweisen, darunter auch Lachen (operational definiert primär durch Brustkorbbewegungen). In Bestätigung von Duncan und Fiske (1977), aber im Widerspruch zu Martin und Gray (1996), wurde „Lachen häufiger bei Frauen aller Altersgruppen beobachtet“ (Adams und Kirkevold 1978: 120); in der geschätzten Altersspanne von 12-17 Jahren trat es am häufigsten auf; und Gäste, die allein waren, lachten nicht unerwartet am seltensten. Adams und Kirkevold bezogen die beobachteten Geschlechtsunterschiede auf „die allgemeine Ansicht, dass Frauen häufiger intimes und geselliges Verhalten zeigen [...] als Männer" (121).

Mit Beginn des einundzwanzigsten Jahrhunderts wuchs das Interesse am Lachen als vokalem Verhalten. Der Neuropsychologe Provine (2000) veröffentlichte das erste Buch zu diesem Thema. Darin betonte er, dass er Anregungen für seine Lachforschung in ethologischen Studien von Tierrufen und Vogelgesang gefunden hatte und sich in seinen Untersuchungen der Feldforschung bediente, wie sie in der Zoologie oder Anthropologie zu 
finden ist. In seinem Buch und in weiteren Artikeln (z.B. 2004) fasste er die Ergebnisse seiner Feldbeobachtungen zusammen, die sich „auf das klassische Gelächter des Typs Ha-ha“ (Provine 2014: 60) beschränkten. Das „lachende Sprechen“ (60) wurde nicht berücksichtigt, weil diese „Mischung aus Lachen und Sprechen unter stärkerer bewusster Kontrolle [steht] und häufiger eingesetzt [wird], wenn man eine Situation entschärfen will“ (Provine 2014: 60). Seine Analysen von insgesamt 1200 heimlich beobachteten Situationen, in denen Menschen an öffentlichen Orten lachten, während sie sich miteinander unterhielten, führten ihn zu der Schlussfolgerung, dass das (HA-HA-) Lachen im Allgemeinen auf nicht humorvolle und banale Äußerungen folgt und typischerweise in Übereinstimmung mit syntaktischen Einheiten auftritt:

Lachen gliedert die Rede. Das Lachen von Sprechern und Hörern trat am Ende von Sätzen oder Satzgefügen auf. Dieser Interpunktionseffekt war außerordentlich stark; nur in weniger als $1 \%$ aller Lachepisoden unterbrach das Lachen die Äußerungen eines Sprechers. Dieser Effekt ist so stark, dass er selbst bei kursorischer Beobachtung von Konversationen bestätigt werden kann (Provine 1993: 296).

Provine (2000) bekannte, dass die Ergebnisse dieser Feldbeobachtungen seine „widerstrebend vollzogene Metamorphose vom Neurowissenschaftler zum Sozialpsychologen auslösten“ (27).

Interessanterweise charakterisierte Provine (2004) das Lachen tatsächlich zugleich als Neurowissenschaftler und Psychologe:

Lachen ist spontan und relativ unzensiert und zeigt deshalb unsere wahren Gefühle. Das Lachen ist, wie auch das Weinen, schwer auf Kommando zu produzieren und deshalb ein aufrichtiges Signal. Wir können nicht absichtlich die Gehirnmechanismen aktivieren, die den affektiven Ausdruck steuern - Lachen ist eine ungeplante Reaktion auf soziale, kognitive und linguistische Hinweisreize (Provine 2004: 216).

Die Psychologin Jo-Ann Bachorowski und ihre Kollegen waren an akustischen Aspekten des Lachens als vokalem Emotionsausdruck interessiert. So untersuchten Smoski und Bachorowski das sogenannte „,,antiphonale، Lachen [...], das während oder unmittelbar im Anschluss an das Lachen des Partners" (Smoski und Bachorowski 2003: 327) auftrat, während gleich- oder gegengeschlechtliche Paare gemeinsam an einer Art von Spiel beteiligt waren. Die Autorinnen definierten Lachen ganz allgemein als „ein Geräusch, das unter altäglichen Umständen als Lachen gelten würde" (331). Ein einzelnes Vorkommen antiphonalen Lachens wurde operational definiert als das Lachen von einem der beiden Probanden, das während des Lachens des anderen auftrat oder innerhalb einer Sekunde nach Beendigung von dessen oder deren Lachen. Smoski und Bachorowski beobachteten unter anderem signifikant mehr antiphonales Lachen bei befreundeten Paaren als bei Paaren, die sich nicht kannten, und bei gemischtgeschlechtlichen Paaren produzierten Frau- 
en mehr antiphonales Lachen als Männer. Sie fanden aber keinen „allgemeinen Hinweis darauf, dass Frauen mehr lachten als Männer" (337) und kamen zu dem Schluss, dass die Funktion des antiphonalen Lachens darin besteht, „gemeinsame positive affektive Erfahrungen“ (327) zu verstärken.

In Anbetracht der unterschiedlichen Methoden, die für die Sammlung von Lachkorpora verwendet wurden, sind Vettin und Todts (2004) Überlegungen wichtig. Sie fanden erste Bestätigungen dafür, dass Lachen, das mit Hilfe von Medien unter experimentellen Bedingungen ausgelöst wurde, sich von konversationellem Lachen in natürlicheren Settings hinsichtlich seiner akustischen Struktur sowie der Länge und Anzahl der Lachzyklen unterscheidet. Zugleich betonten sie, dass es zur Überprüfung dieses Befundes weiterer Untersuchungen bedarf.

Das Jahr 2007 erwies sich als sehr stimulierend für die Lachforschung. Der Linguist Wallace Chafe veröffentlichte sein Buch The Importance of Not Being Earnest: The Feeling Behind Laughter and Humor, und ein internationaler Workshop über die Phonetik des Lachens wurde in Saarbrücken mit einem Einführungsvortrag von Chafe eröffnet. Besonders hervorzuheben ist Chafes systematische Darstellung der Phonetik des Lachens im ersten Teil des genannten Buches (Chafe 2007: 17-58). Er legt darin eine der aufschlussreichsten Zusammenfassungen der akustischen und phonetischen Eigenschaften des Lachens vor, die wir bisher gefunden haben. Unter anderem weist er darauf hin, dass das, was im Mund passiert, für das Lachen weniger wichtig ist als für das Sprechen. So sind die Vokale beim Lachen unklarer als beim Sprechen, weil sich die Stimmbänder während der Stimmgebung beim Lachen nicht so vollständig schließen wie dies beim Sprechen der Fall ist.

Zusammenfassend zeigt der historische Literaturüberblick, dass die empirische Erforschung des vokalen Lachens in dialogischer Interaktion zwar vereinzelt zu interessanten Ergebnissen geführt hat, dass dieses Thema bisher jedoch keine große Rolle in der psychologischen Forschung gespielt hat. Deutlich wurde auch, dass dieses komplexe Verhalten bisher nur gelegentlich interdisziplinär untersucht wurde; das betrifft sowohl phonetisches und linguistisches Wissen um die Vokalstruktur verschiedener Arten des Lachens, biologisches und neurologisches Wissen wie auch soziologische und psychologische Aspekte. Das Interesse an der Form und Funktion des Lachens in dialogischer Interaktion, das bereits in den 1920er Jahren begonnen hatte, bestimmt auch in neuerer Zeit die psychologische Forschung und hat zu Überschneidungen mit konversationsanalytischen Arbeiten geführt. Empirische Untersuchungen berücksichtigen den Einfluss von sozialen Auslösern des Lachens, von Geschlechtsunterschieden und von der Wahl zwischen experimentellen und Feldbeobachtungsmethoden. Dennoch hat sich bisher keine empirisch orientierte Tradition der Lachforschung innerhalb der Psychologie entwickelt. 


\section{Definitionen, Merkmale und Funktionen des vokalen Lachens}

Angesichts des Mangels an übereinstimmenden Definitionen des vokalen Lachens erscheint die bereits oben erwähnte allgemeine Definition von Bachorowski, Smoski und Owren (2001) ein sinnvoller Ausgangspunkt zu sein: „Jedes hörbare Geräusch, das ein normaler Mensch unter alltäglichen Bedingungen als Lachen bezeichnen würde" (1582). Provine (2000; vgl. auch Jefferson, Sacks und Schegloff, 1984: 18) hat ein weiteres Merkmal hinzugefügt: Lachen ist „der Inbegriff des menschlichen sozialen Signals“ und hat deshalb „mit Beziehungen“ (43) zu tun. Glenn (2003: 14) hat aus konversationsanalytischer Perspektive festgelegt: „Lachen ist sowohl Ausdrucksform eines einzelnen Menschen wie einer Gruppe von Menschen", und Hopper (1992: 179) hat als wesentliches Merkmal des Lachens das Ausatmen bestimmt: "Lachen ist eine Art unfreiwilliges vokales Ausatmen, das Vergnügen, Albernheit oder etwas Unerwartetes signalisiert“. Hierzu ist kritisch anzumerken, dass es keinerlei Hinweise darauf gibt, dass das Lachen unfreiwillig sein muss oder dass es auf die von Hopper aufgezählten Funktionen beschränkt sein muss. Chafe (2007: 22ff.) hat noch „inhalatory pulses“ (22ff.) als charakteristisch für das Lachen hinzugefügt. Und der Phonetiker Trouvain (2003) hat das Lachen als „eine alltägliche, spezifisch menschliche, affektive, nonverbale Vokalisation“ (2793) beschrieben. Aber diese Charakterisierung ist zu ungenau: Einerseits schließt sie zu viel ein, nämlich nicht nur das Lachen sondern auch das Weinen; andererseits scheint sie diejenige Art des Lachens auszuschließen, die zugleich mit dem Sprechen auftritt und die wir als suprasegmental bezeichnen, da es sich hier um „einen vokalen Effekt [handelt], der über mehr als ein +Segment anhält" (Crystal 1997: 438). Jedoch hatte Trouvain (2001) bereits diese von ihm "Sprech-Lachen“ (634) genannte Variante in einer Pilotstudie mit einem Korpus spontaner Dialoge analysiert. Dabei stellte er fest, dass das Sprech-Lachen häufig auftrat (60\% allen Lachens), zugleich aber nicht verlässlich identifiziert und vom Sprech-Lächeln abgegrenzt werden konnte. Trouvain (2014) betonte die Komplexität und die phonetische Variabilität des Lachens und unterschied "gesangähnliches Lachen" (599), das stimmhaft oder stimmlos sein kann, vom Sprech-Lachen und vom gemeinsamen, das heißt interaktiven Lachen.

Sowohl der historische Überblick zur Lachforschung (insbesondere Sully 1902/2012 und Hayworth 1928) als auch die alltägliche Erfahrung legen nahe, dass die Untersuchung des Lachens nicht von der Annahme ausgehen kann, dass das Lachen notwendig angenehme Gefühle ausdrückt, ebenso wenig wie die psychologische Untersuchung des Weinens von der Annahme ausgehen kann, dass das Weinen immer mit traurigen Gefühlen einhergeht. Provine (2004) hat das Lachen aus evolutionsbiologischer Perspektive als ,instinktive, ansteckende, stereotypische, unbewusst gesteuerte Vokalisierung spielerischer sozialer Interaktion“ (215) definiert. Abgesehen davon, dass die genannten Begriffe alle eine gewisse Mehrdeutigkeit in sich tragen (vgl. etwa die Kontroverse über den Begriff „stereotyp“ in 
Provine (2014: 271, Fußnote 18), ist keine dieser Eigenschaften unserer Meinung nach ein notwendiges Merkmal des Lachens - besonders nicht des komplex motivierten Lachens von Erwachsenen. Mit anderen Worten, es gibt das Lachen, das nicht instinktiv, nicht ansteckend, nicht stereotyp, nicht unbewusst gesteuert ist und keine Vokalisierung einer spielerischen sozialen Interaktion - eine Position, die für unsere eigene Forschung wichtig ist.

\section{Empirische Untersuchungsmethoden}

Der größte Teil der frühen Lachforschung hat sich mit dem HA-HA-Lachen beschäftigt, das nicht - wie das suprasegmentale Lachen - gleichzeitig mit verbalen Äußerungen auftritt. Diese Art des Lachens findet sich auch stereotypisch in der schriftlichen Darstellung des Lachverhaltens. Das folgende literarische Beispiel ist aus dem Roman von Roger Martin du Gards Die Thibaults (1928/2003: 83): „Er hatte eine etwas unnatürliche Art zu lachen: Er warf den Kopf nach hinten, so daß seine Augäpfel an den Lidrand rollten, und dann ließ er nacheinander drei etwas gezierte ,ha' fallen: ,Ha! ha! ha!"“

Tatsächlich zeigen akustische Analysen, dass das HA-HA-Lachen mit ganz verschiedenen Lauten gebildet werden kann, die z.B. als he, ha, hm, ho oder einfach als stimmloses behauchtes $h$ transkribiert werden können. Erst im Laufe der Zeit hat sich ein wachsendes Interesse am suprasegmentalen Lachen herausgebildet. So haben etwa Nwokah, Hsu, Davies und Fogel (1999) berichtet, dass Mütter in der Interaktion mit ihren Kindern in $50 \%$ der Fälle während des Sprechens lachen.

Beide Arten des Lachens - HA-HA-Lachen und suprasegmentales Lachen - können in Einheiten von Calls und Bouts analysiert werden (Bachorowski, Smoski, und Owren 2001). Calls (C) sind einzelne Pulse, die einer Sprechsilbe vergleichbar sind, Bouts (B) bestehen aus einer Sequenz von Calls, die durch Pausen einer bestimmten Mindestdauer begrenzt wird. Entsprechend werden Calls/Bout (C/B), Calls/Silbe (C/Sil) und Calls/Sekunde (C/Sek) zu wichtigen relativen Messwerten für die Bestimmung der Länge, Häufigkeit und Dauer des Lachens. Maße wie diese sind für eine quantitative Analyse des Lachens nötig und ermöglichen den Vergleich verschiedener Datensätze.

Calls und Bouts als phonetische Einheiten des Lachens sind zu unterscheiden von Provines sogenannten "laugh episodes" (Provine 2000: 26), die er folgendermaßen definierte: „Eine Lachepisode besteht aus dem [verbalen] Kommentar, der dem Lachen direkt vorausgeht, und dem gesamten Lachen, das innerhalb einer Sekunde nach dem Beginn des Lachens auftritt“ (26). Wie oben erwähnt, beschränkte sich Provine auf das HA-HALachen. Mit Hilfe der Lachepisoden überprüfte er, wie stark der Zusammenhang zwischen Lachen und Humor ist. Er stellte fest, dass „nur 10-15\% der dem Lachen vorausgehenden Kommentare annähernd komisch waren“ 
(Provine 2004: 215), während die meisten Kommentare einfach banal, auf jeden Fall aber nicht komisch waren. In einer seiner Feldstudien schickte Provine Studenten, lediglich mit einem Klemmbrett für Notizen ausgestattet, auf die Straßen von Baltimore, um Passanten, die sich unterhielten, aus einer gewissen Entfernung heimlich zu beobachten. Dem Vorteil einer solchen Feldbeobachtung, ihrer ökologischen Validität, steht der Nachteil gegenüber, dass derartige Beobachtungen keine Überprüfung ihrer Zuverlässigkeit zulassen.

Ein weiteres Problem der empirischen Lachforschung betrifft die Vergleichbarkeit von Ergebnissen, die unter unterschiedlichen Bedingungen der Datenerhebung ermittelt wurden. In einigen psychologischen Studien wurde das Lachen unter experimentellen Bedingungen durch den Einsatz verschiedener Medien humoristischen Inhalts ausgelöst (z.B. Chapman 1973a) oder durch bestimmte Aufgaben, die Probanden paarweise durchführen sollten (z.B. Smoski und Bachorowski 2003). In anderen Studien wurden Paare in ein Aufnahmestudio gebracht und gebeten, sich miteinander über irgendein für sie interessantes Thema zu unterhalten (z.B. Duncan und Fiske 1977), während sie offen oder verdeckt gefilmt wurden. Noch andere Forscher führten Feldbeobachtungen durch (z.B. Provine 1993). Wie oben erwähnt (vgl. Abschnitt 3) haben Vettin und Todt (2004) auf das Problem der möglichen Unvergleichbarkeit der Daten hingewiesen. Weiterhin wirft die Verwendung fremder statt eigener Korpora Fragen bezüglich der Reliabilität und Validität der Transkriptionen auf. Von solchen Problemen haben etwa Trouvain und Truong (2012) in ihrer Analyse von sechs fremden Korpora berichtet. Sie fanden heraus, dass HA-HA-Lachen in diesen Korpora immer transkribiert worden war, während suprasegmentales Lachen in zwei der Korpora nicht enthalten war. Die Vergleichbarkeit der sechs Korpora wurde dadurch stark eingeschränkt.

\section{Untersuchungen des Lachens von O'Connell und Kowal}

Unsere eigene Beschäftigung mit dem Lachen begann mit der Frage nach der Verallgemeinerbarkeit der Ergebnisse von Provine, dem prominentesten Vertreter der gegenwärtigen psychologischen Lachforschung - und zum Teil wegen Zweifeln an der grundsätzlicher Plausibilität seiner Verallgemeinerungen. Die klassische entwicklungspsychologische Untersuchung von Washburn (1929) hatte zwar deutliche Hinweise auf das Vorhandensein von Stereotypien im Lachen von Kleinkindern gegeben. Aber wir gingen davon aus, dass sich das Lachverhalten von Erwachsenen aufgrund vielfältiger Lernerfahrungen und kultureller Einflüsse im Laufe ihres Lebens in Form und Funktion erheblich verändern kann.

Aus mehreren Gründen wählten wir Lachkorpora aus Medieninterviews und aus Spielfilmen: Beide Kontexte bieten Beispiele für den genuin kommunikativen Gebrauch des Lachens; von Sprecherinnen oder Sprechern wird in diesen Kontexten weiterhin erwartet, dass sie relativ deutlich arti- 
kulieren; und öffentlich verfügbare Dialoge liefern in aller Regel Daten von guter akustischer Qualität, eine Voraussetzung für reliable und valide Analysen des vokalen Lachens. Zugleich stellt sich bei derartigen Daten nicht das ethische Problem verdeckter Tonband- oder Videoaufnahmen.

\subsection{Das Fernsehinterview „Zur Person“ von Günter Gaus mit Hannah Arendt}

In unserer Studie (O'Connell und Kowal 1998) des Interviews von G. Gaus mit H. Arendt aus dem Jahr 1964 lag der thematische Schwerpunkt nicht auf dem Lachverhalten sondern auf der „Mündlichkeit und Schriftlichkeit in öffentlichen Diskursen“ (543). Um dieses Thema empirisch zu behandeln, verglichen wir unser eigenes Transkript des gesamten Interviews mit der in mehreren Publikationen abgedruckten Version desselben Interviews. Lachen wurde in unseren Analysen mit Husten, Räuspern und hörbarem Seufzen in der Kategorie „paralinguistische Phänomene“ (553) zusammengefasst und nicht transkribiert sondern lediglich beschrieben (z.B. LACHEN, RÄUSPERN). Insgesamt traten paralinguistische Phänomene 48-mal auf, 33-mal bei Arendt (in 24 Fällen handelte es sich um Lachen) und 15-mal bei Gaus (er lachte 4-mal).

Es zeigte sich, dass für die Publikation des Interviews alle 48 Vorkommen paralinguistischer Phänomene getilgt wurden. Offenbar sind die Herausgeber davon ausgegangen, dass das Lachen kein kommunikatives Mittel ist, das zum Verständnis der intendierten Bedeutung von Äußerungen beitragen kann. Beispiel 1 zeigt einen Interviewausschnitt im Transkript und in einer publizierten Version, in dem Hannah Arendt (HA) während ihrer Antwort auf eine Frage von Günter Gaus (GG) lacht. Die gesprochenen Äußerungen, die in der Druckversion veröffentlicht wurden, sind im Transkript in Fettdruck markiert.

Beispiel 1 (nach O’Connell und Kowal 1998: 558)

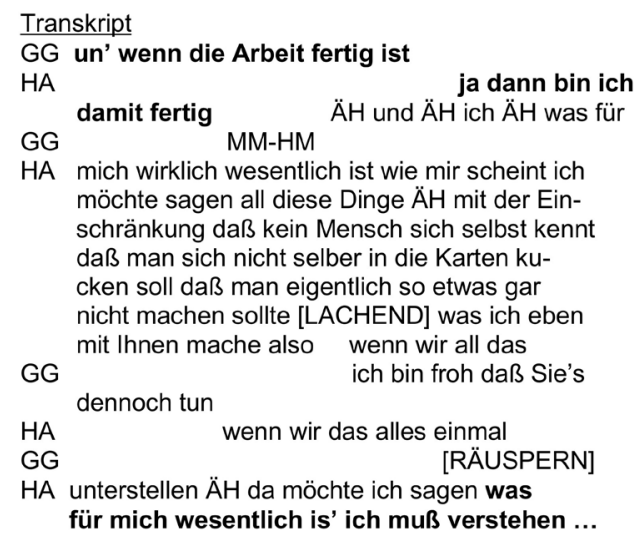

Publikation

GAUS: Und wenn die Arbeit fertig ist? ARENDT: Ja, dann bin ich damit fertig. Wissen Sie, wesentlich ist für mich: Ich muß verstehen. (Arendt 1996: 46) 
Das Beispiel zeigt die beachtliche Verringerung der Anzahl gesprochener Silben in der Publikation im Vergleich zum Transkript (30<136 Silben). Dadurch gehen die dialogische „Aktgeschichte“ (Bühler 1934/1982: 56) der allmählichen Verfertigung von Arendts Antwort verloren und die Hörerrückmeldungen von Gaus. Mit diesen Auslassungen wird es für den Leser oder die Leserin kaum möglich, Arendts zögerlich formulierte Haltung zu ihrer eigenen spontanen Selbstenthüllung nachzuvollziehen. Ihre erste Antwort (,ja dann bin ich damit fertig") auf seine Frage trat übrigens erst nach einer längeren Pause von 0,98 Sekunden auf und wurde relativ langsam artikuliert. Offenbar sah Hannah Arendt zugleich die Notwendigkeit, aber auch die Möglichkeit, ihre direkte Antwort zu differenzieren, indem sie ihr persönliches Motiv für „die Arbeit“ hinzufügte - nämlich nicht „eine Wirkung auch in der Breite" zu erzielen, wie Gaus es in seinem vorausgegangenen Redebeitrag vorgeschlagen hatte, sondern: „Ich muß verstehen ÄH zu diesem Verstehen gehört bei mir auch das Schreiben". Zugleich scheint Arendts Lachen eine gewisse Verlegenheit darüber auszudrücken, dass sie sich, entgegen ihrer eigenen Überzeugung, durch Gaus dazu verleiten lässt, etwas von ihrer Person preiszugeben. Beispiel 1 (ebenso wie andere Teile diese Interviews) vermittelt zudem einen Einblick davon, wie Gaus dialogisch seine InterviewpartnerInnen in der Sendung „Zur Person“ dazu bringt, über sich selbst zu reden: „Mein Partner soll nicht mit mir argumentieren, sondern von sich erzählen“ (Gaus 1964: 10).

\subsection{Fernseh- und Radiointerviews mit Hillary Clinton und Bill Clinton}

Unsere weiteren Studien konzentrierten sich auf das Lachen selbst. Im Anschluss an die Veröffentlichung ihrer jeweiligen Memoiren $(\mathrm{H}$. Clinton 2003; B. Clinton 2004) gaben Hillary Clinton und Bill Clinton eine Reihe von Interviews, in denen sie auch für ihre Bücher warben. Diese Folge von Rundfunk- und Fernsehinterviews erlaubte uns durch die methodische Kombination von Feldbeobachtung mit einer quasi-experimentellen Komponente eine Reihe von quantitativen und qualitativen Vergleichen durchzuführen. Das Korpus von H. Clintons Interviews (O'Connell und Kowal 2004) umfasste 4 Fernseh- und 2 Radiointerviews; das Korpus von B. Clintons Interviews (O'Connell und Kowal 2005) umfasste 11 Fernseh- und 2 Radiointerviews. Fünf der InterviewerInnen befragten beide Clintons. Während die Studie über H. Clinton - wegen ihres Pilotcharakters - weitgehend auf eine Analyse von Calls und Bouts des HA-HA-Lachens beschränkt war, umfasste die Studie von B. Clinton beide Arten des Lachens (HA-HA-Lachen und suprasegmentales Lachen). In beiden Studien wurde das auditiv wahrnehmbare Vorkommen von Lachen der Clintons und ihrer Interviewerlnnen mit Hilfe der PRAAT-Software akustisch analysiert. Die quantitativen Analysen umfassten Anzahl und Dauer der Bouts (B), die Anzahl der Calls (C), Silben/Bout (Sil/B) und Silben/Call (Sil/C). In beiden Studien wurden außerdem die Themen analysiert, bei denen das Lachen auftrat. 


\subsubsection{Hillary Clinton}

H. Clintons Interviews eigneten sich gut für eine Pilotstudie des Lachens. In ihren Memoiren (Clinton 2003) hatte sie ihren Vater für ihr eigenes auffälliges, lautes Lachen verantwortlich gemacht: „Ich erbte dieses Lachen, dieses herzhaft schallende Gelächter, bei dem sich die Leute im Restaurant zu uns umdrehen und Katzen auf der Stelle Reißaus nehmen" (4). Außerdem überwog in den Interviews das HA-HA-Lachen, das in den instrumentellen Analysen eindeutiger zu identifizieren war. Unserer Studie (O'Connell und Kowal 2004) lag die allgemeine Annahme zugrunde, dass Gesprächspartnerlnnen durch ihr Lachen vor allem ihre eigene, oft verbal nicht formulierte Perspektive auf das gerade behandelte Gesprächsthema ausdrücken:

Eine Perspektive ist einfach eine Position, von der aus eine Person oder eine Gruppe etwas (Gegenstände, Personen oder Ereignisse) betrachtet und ihre Ansichten kommuniziert (Graumann \& Kallmeyer 2002: 1). Eine grundlegende Annahme der auf den Sprachgebrauch angewandten Theorie der Perspektivität ist, dass jede Äußerung, die ein Sprecher oder eine Sprecherin als ,Autor' (Goffman 1981: 226) tätigt, perspektiviert ist, d.h. eine persönliche Perspektive einbezieht (O'Connell und Kowal 2004: 465).

Folglich betrachteten wir H. Clintons Lachen als ein wichtiges Mittel, um ihre Einstellung zu den angesprochenen Interviewthemen indirekt, nämlich paralinguistisch, auszudrücken und damit eine explizite (verbale) Stellungnahme zu vermeiden oder hinauszuzögern. Auf dem Hintergrund der Behauptung von Clayman und Heritage (2002), dass Interviewerlnnen in Medien typischerweise Neutralität praktizieren, erwarteten wir weiterhin, dass sie nur selten lachen. Wäre dies der Fall, so stellte sich allerdings die Frage, ob bzw. wie H. Clinton „auf freiwilliger Basis“" (Jefferson 1979: 81) lacht, das heißt nicht reaktiv, sondern vorwiegend aus eigener Initiative.

Quantitative Analysen zeigten, dass $\mathrm{H}$. Clinton in allen sechs Interviews lacht, aber in den Fernsehinterviews relativ häufiger als in den Radiointerviews. Clintons HA-HA-Lachen trat häufiger während der Redebeiträge der Interviewerlnnen auf als während ihrer eigenen Redebeiträge. Eine Analyse der initialen, finalen und medialen Positionen ihres Lachens in Relation zu artikulatorischen Phrasen (von Pausen unterbrochene Sequenzen von Silben) zeigte, dass lediglich ein Drittel in finaler Position auftrat, ein Ergebnis, das deutlich von Provines Interpunktionseffekt abweicht. In allen vier Fernsehinterviews, jedoch in keinem der beiden Radiointerviews, lachte $\mathrm{H}$. Clinton im Zusammenhang mit ihrer angekündigten Präsidentschaftskandidatur, ein Thema, das sowohl in Fernseh- als auch in Radiointerviews diskutiert wurde. Ihre Kandidatur war zugleich das Thema, bei dem sie am häufigsten lachte, wobei nur einer ihrer Interviewer (der Komiker David Letterman) gemeinsam mit ihr über dieses Thema lachte. 
Das nachfolgende Beispiel haben wir dem Fernsehinterview von $\mathrm{H}$. Clinton (HC) mit Katie Couric (KC) über H. Clintons Kandidatur (O'Connell und Kowal 2004) entnommen. Es enthält zusätzlich einen Redebeitrag von H. Clinton (fettgedruckt), der aus dem unveröffentlichten Transkript des Interviews hinzugefügt wurde.

Beispiel 2

KC you have said Senator Clinton you will not run for president in two thousand four tsk what if your party drafted you

$\mathrm{HC}$

have very oh no you

(LAUGHING) that's not gonna happen we

what if it did what if they came to you and said Senator Hillary Clinton you are the only person in our view who can beat president Bush what would you do

$\mathrm{HC}$

In diesem Beispiel wird die erste verneinende Antwort von $\mathrm{H}$. Clinton, die anschließend mehrfach wiederholt wird, mit einem Lach-Bout (als LACHEN im Transkript notiert) eingeleitet. Aber weder Courics Frage noch H. Clintons Antwort ist erkennbar komisch. Vielmehr vermeidet $\mathrm{H}$. Clinton durch ihr Lachen, auf Courics Frage sofort explizit, das heißt verbal, zu reagieren. Auch ihr anschließender Kommentar („that's not gonna happen“) bleibt meisterhaft allgemein und deshalb wenig informativ. Insgesamt war in ihren Fernsehinterviews zu beobachten, dass H. Clinton häufig (gemessen in Bouts) in Reaktion auf Fragen der Interviewerlnnen, die weder scherzhaft gemeint noch humorvoll waren, diese Vermeidungsstrategie mittels Lachen anwendete. Mit ihrem Lachen „auf freiwilliger Basis“ (Jefferson 1979: 81) scheint $\mathrm{H}$. Clinton zugleich implizit anzudeuten, dass sie die gestellten Frage nicht ganz ernst nimmt. Während eine solche Interpretation sich zeitnah (proximal) auf die sprachlichen Äußerungen der Interviewerlnnen bezieht, würde Provine (2014) das Lachen aus evolutionärer Perspektive weniger als Kommentar zu einer Äußerung, sondern als Kommentar zu der sich äußernden Person interpretieren. Nach Provine kann Lachen eine

reichhaltige Quelle für Informationen über komplexe zwischenmenschliche Beziehungen [sein]. Gelächter „lesen“ zu lernen, ist vor allem deshalb nützlich, weil es sich um eine unwillkürliche Verhaltensweise handelt, die sich nur schwer vortäuschen lässt. Sie gibt also unzensiert und ehrlich wieder, was Menschen übereinander [...] denken (71; Kursive Hervorhebung nachträglich von den Verfassern hinzugefügt).

Schließlich haben JournalistInnen an $\mathrm{H}$. Clintons Lachen wiederholt eine gewisse Künstlichkeit in ihrer öffentlichen Selbstdarstellung festgestellt. So betonte Rich (30. September 2007, WK 12) auf der Meinungsseite der New York Times den automatenähnlichen Charakter und die implizite Unaufrichtigkeit des rauen und explosiven Lachens von $\mathrm{H}$. Clinton: 
Dann war dieses Lachen.... .

Jetzt brach Mrs. Clinton in ein Lachen aus, das die Spontaneität eines Weckeralarms hatte. Um diesen Tic ins Lächerliche zu ziehen, malte sich „The Daily Show“ eine Roboterstimme aus, die im Kopf der Kandidatin meldet: „Iustige Bemerkung gefunden - vorbereiten zur Ausgabe des Lachens $[\ldots]^{\prime \prime}$.

Und Flores (27. Juni 2014) beschrieb H. Clintons nervöses, „unbehagliches Lachen" als gewohnheitsmäßige Reaktion oder Tic in Reaktion auf Interviewfragen - als ein befremdlich und bizarr wirkendes Lachen. Diese Bewertungen stimmen mit den oben (Abschnitt 2.2) zitierten negativen Einstellungen gegenüber „unangemessenem“ Lachen von Frauen überein. Eine Interpretation von $\mathrm{H}$. Clintons Lachen als gewohnheitsmäßige Reaktion würde tatsächlich eine gewisse Indifferenz sowohl gegenüber den Fragen der Interviewerlnnen wie auch ihrer Person implizieren.

Romaniuk (2009) hat sich aus konversationsanalytischer Sicht mit der Praxis von Interviewten beschäftigt, vor einer ernsthaften Antwort auf eine Frage zunächst zu lachen, und sich dabei auch speziell auf $\mathrm{H}$. Clinton bezogen:

Das Reagieren auf den gerade produzierten Gesprächsbeitrag des Interviewers erlaubt HRC [Hilary Rodham Clinton] implizite Kommentare zum Gesagten noch vor der Beendigung der gestellten Frage und vor ihrer eigentlichen Antwort. Auf diese Weise demonstriert HRC, wie das Lachen als interaktive Ressource von Interviewten in Nachrichteninterviews des Fernsehens benutzt werden kann (Romaniuk 2009: 42).

\subsubsection{Bill Clinton}

Mit demselben perspektivischen Ansatz gingen wir in unserer Studie über B. Clinton (O'Connell und Kowal 2005) davon aus, dass das Lachen „in einer absichtlichen, durchdachten und rhetorischen Weise gebraucht werden kann“ - eine Annahme, die nicht notwendig eine direkte, bewusste Verhaltenskontrolle (278) unterstellt. Quantitative Analysen zeigten, dass B. Clinton in 10 von 12 Interviews zwar mindestens einmal lachte, dass sein Lachen aber insgesamt selten auftrat und dass es keine Unterschiede in dessen Häufigkeit zwischen Fernseh- und Radiointerviews gab. Dabei handelte es sich häufiger um suprasegmentales Lachen als um HA-HA-Lachen.

In einigen Hinsichten stimmten die Resultate für B. Clinton mit denen von H. Clinton überein: Wir fanden für beide keinen Hinweis auf Provines (1993: 296) „extrem starken“ Interpunktionseffekt, da wir uns nicht auf HA-HALachen beschränkt hatten. Das Lachen der Clintons folgte zudem in der Regel weder auf banale Kommentare (Provine 2004:215) noch auf humorvoll gemeinte Aussagen (Chafe 2007). Und das Thema, das bei B. Clinton am relativ häufigsten lachend behandelt wurde, war ebenfalls $\mathrm{H}$. Clintons Kandidatur für die US-Präsidentschaft. Ein weiteres Thema war die öffentliche Kritik an seinem Privatleben, wie Beispiel 3 weiter unten zeigt. Es scheint, dass B. Clinton in diesen Fällen Lachen als ein rhetorisches Stil- 
mittel benutzte, um die Bedeutung von problematischen Themen zu entschärfen bzw. als für inn erledigt zu markieren. Sein Gebrauch des Lachens scheint sich in dieser Funktion stark von einem „relativ unzensierten und so unsere wahren Gefühle zeigenden“ Lachen (Provine 2004:216) zu unterscheiden. Im Gegenteil scheinen B. Clintons „wahre“ Gefühle durch sein Lachen tatsächlich verdeckt zu werden.

Unterschiede zwischen $\mathrm{H}$. und B. Clinton traten im Hinblick auf zwei Messgrößen auf. (1) Während $\mathrm{H}$. Clinton üblicherweise eine Äußerung der Interviewerlnnen mit HA-HA-Lachen begleitete, reagierte B. Clinton eher auf das, was er selbst sagte, mit suprasegmentalem Lachen. In diesem Sinne blieb H. Clintons Lachen oft semantisch undurchsichtig auf die Äußerungen der InterviewerInnen bezogen, während B. Clintons Lachen im Allgemeinen semantisch eher transparent auf eigene Äußerungen bezogen war. Ein ähnliches geschlechtsspezifisches Ergebnis beobachtete Dreher (1982; zitiert in Kotthoff 1996: 127) für Unterhaltungen zwischen StudentInnen: Das Lachen von Studenten war im Allgemeinen selbstreferenziell, während das Lachen der Studentinnen sich generell auf die Äußerungen anderer bezog. Dreher fand auch, dass Studentinnen doppelt so oft lachten wie Studenten. (2) Häufigkeit des HA-HALachens. Ein Vergleich der Häufigkeit von B. Clintons HA-HA-Lachen mit $\mathrm{H}$. Clintons HA-HA-Lachen (in Silben/Call) in den Interviews mit denselben InterviewerInnen zeigte, dass $\mathrm{H}$. Clinton in allen fünf Interviews häufiger lachte als B. Clinton. Dieses Ergebnis bestätigt sowohl Dreher als auch Provine (1993: 295) über den Einfluss des sozialen Geschlechts auf die Bereitschaft zu lachen.

Jefferson (1984) untersuchte das Lachen von Personen, die lachend über Probleme sprechen. Sie stellte fest, dass ein Zuhörer oder eine Zuhörerin in solchen Situationen "nicht lacht, sondern erkennbar ernsthaft reagiert" (346). Unsere Studie über B. Clintons Lachen (O'Connell und Kowal 2005) bestätigte Jeffersons Beobachtungen im Hinblick auf die Interviewerlnnen. In Beispiel 3, in dem der Interviewer Charlie Rose (CR) ein ernstes und heikles Thema anspricht, antwortet B. Clinton (BC) lachend - mit HA-HALachen (als HU notiert) und suprasegmentalem Lachen (durch Unterstreichung notiert).

Der in Beispiel 3 nicht enthaltene nächste Redebeitrag von Charlie Rose zeigt, dass er nicht in das Lachen von Clinton einstimmte, sondern unverzüglich zur nächsten Frage überging.

Beispiel 3

$\mathrm{CR}$ well there was also this you were gettin' beat up so bad at home that you were anxious to get to the office

$\mathrm{BC}$ that's right I said that uh yeah that's ri-hi-hi-hight HU HU HU HU I probably was more attentive to my work for several mohonths just because I didn't want to have to attend to anything else (286) 


\subsection{Der Film „Der dritte Mann“ in der englischsprachigen (1949) und in der deutschsprachigen (1962) Version}

Wir gingen auch in unserer nächsten Studie davon aus, dass das Lachen in Dialogen „viele Zwecke erfüllt. Es kann vergnügt, amüsiert, wohlwollend und ansteckend sein, aber auch zynisch, gemein, unangemessen, arrogant und verächtlich" (O'Connell und Kowal 2006: 306). Einen extremen Fall für die letztere Variante fanden wir in dem Film The Third Man, einem klassischen film noir von Carol Reed, Alexander Korda und David O. Selznick aus dem Jahr 1949. Die Verfügbarkeit des englischen Originals und der deutschen Synchronisation des Films erlaubten auch einen Vergleich des Lachverhaltens der SchauspielerInnen mit dem der Synchronsprecherlnnen. Da zudem umfangreiche schriftliche Hintergrundinformationen zu diesem Film vorlagen, konnten wir mit dieser Studie zusätzlich das Thema Schriftlichkeit vs. Mündlichkeit weiter verfolgen, das wir in O'Connell und Kowal (1998) behandelt hatten (für mehr Informationen zu diesem Thema siehe O'Connell und Kowal 2006: 307f. und passim).

Das Korpus für die empirischen Analysen des vokalen Lachens wurde aus eigenen Transkripten von Audiokassetten der englisch- und der deutschsprachigen Filmversionen zusammengestellt. Die Analysen erfolgten größtenteils mit denselben Methoden wie in O'Connell und Kowal (2005). Zusätzlich wurden die folgenden funktionalen Kategorien des Lachens auf Basis des verbalen und des situativen Kontextes unterschieden: zynisches, verlegenes, humorvolles, heuchlerisches, ironisches, verlogenes und sympathisches Lachen.

Im englischen Original trat Lachen in insgesamt 34 Bouts auf und in der synchronisierten deutschen Version in 48 Bouts. Insgesamt unterschied sich die Häufigkeit der beiden Arten des Lachens (HA-HA-Lachen vs. suprasegmentales Lachen) in den beiden Filmversionen nicht. Dagegen lachten die SynchronsprecherInnen nur in $50 \%$ der Fälle an der gleichen Stelle der fortlaufenden Filmunterhaltung wie die Schauspielerlnnen, das heißt das Lachen der SynchronsprecherInnen war in diesem Sinne keine exakte Kopie. Die folgenden Beispiele $4 \mathrm{a}$ und $4 \mathrm{~b}$ geben die Transkripte von zwei Äußerungen des „dritten Mannes“ Harry Lime im englischsprachigen Original und in der deutschen Synchronfassung wieder. Beide Beispiele entstammen der berühmten Szene am Riesenrad im Wiener Prater, in der Lime und sein alter Freund Holly Martins sich gegen Ende des Films zum ersten Mal begegnen (HA-HA-Lachen ist durch HU notiert, suprasegmentales Lachen durch Unterstreichung).

Ein Vergleich der Beispiele 4a und 4b zeigt, dass das Lachen in den beiden Versionen sowohl bezüglich der Länge als auch der Art variiert - Abweichungen, die auch für andere Vorkommen des Lachens in der originalen und der synchronisierten Filmversion zu beobachten sind. Synchronsprecherlnnen sind also in diesem Fall in den von Goffman (1981:226) geprägten Begriffen eher „AutorInnen“ (authors) bzw. „UrheberInnen“ (principals) 
ihres Lachens als lediglich „VertonerInnen“ (animators) des Lachens eines vorgegebenen Modells.

Beispiel 4a

Lime hello old ma-han h-how are you

Lime HU Tag alter Junge wie geht's denn

Beispiel 4b

Lime what can I do old man I'm dead HU aren't I

Lime MM was kann ich tun mein Junge ich bin tot

Weitere Analysen bestätigten, dass die Vokale des HA-HA-Lachens nicht so artikuliert werden, wie es inre stereotypisierende Notation (HA HA) nahelegt. Ein Beispiel ist das als „UH I'YO“ (O'Connell und Kowal 2006: 309) transkribierte Beispiel eines HA-HA-Lachens. Interessanterweise scheint es eine Überschneidung in der lautlichen Form und im Gebrauch von HA-HA-Lachen und Interjektionen zu geben. So kommt das erwähnte Beispiel dem sehr nahe, was Rhodes (1992: 222) als „,wilde“ Interjektionen“ den „zahmen“ bzw. konventionellen Interjektionen gegenüber gestellt hat. Dort wo HA-HA-Lachen und suprasegmentales Lachen innerhalb desselben Bout erzeugt wurden, trat das HA-HA-Lachen typischerweise vor dem suprasegmentale Lachen auf (O'Connell und Kowal 2004). Diese Beobachtung stimmt mit Befunden aus der Interjektionsstudie von O'Connell und Kowal (2010: 297) überein: In einem Korpus von 399 Interjektionen aus der Verfilmung von Jane Austins Pride and Prejudice traten $94 \%$ aller Interjektionen vor dem folgenden Redebeitrag auf. In diesem Zusammenhang ist von historischen Interesse, dass das MerriamWebster's Collegiate Dictionary (11. Auflage 2003) ha-ha als eine Interjektion auflistet, die „benutzt wird, um Amüsement bzw. Spott auszudrücken (560)" und deren Geschichte als Teil des geschriebenen Englisch bis ins frühe zwölfte Jahrhundert zurückgeht und dem Altenglischen ha-ha entstammt.

Die Analysen des Lachens in den verschiedenen funktionalen Kategorien des Lachens zeigten, dass "harmloses“ (scherzhaftes, ironisches, umgängliches und verlegenes) Lachen insgesamt in beiden Filmversionen genauso oft vorkam wie "negatives“ (zynisches, heuchlerisches und verletzendes) Lachen (O'Connell und Kowal 2006: 324). Jedoch gingen alle 16 Vorkommen der letzteren Kategorie im englischen Original und alle 25 Vorkommen in der deutschen Synchronisation allein auf das Konto der „Schurken“ Kurz, Lime und Popescu. Dieses Ergebnis bestätigt wiederum die perspektivische Natur des Lachens und seinen Gebrauch als rhetorisches Mittel. Das Lachen ist hier kaum als ,instinktive, ansteckende, stereotypische, unbewusst gesteuerte" Vokalisierung zu bezeichnen, wie Provine (2004: 215) es beschrieben hat. Als die dominante Art des Lachens in diesem Korpus ist das suprasegmentale Lachen auch kaum als „kurioser Zwitter" (Provine 2004: 216) zu bezeichnen. 
6.4 Mrs. Bennets Lachen in der BBC Fernsehserie „Pride and Prejudice“ (1995)

In ihrer phonetischen Studie des Emotionsausdrucks haben Pompino-Marschall, O'Connell und Kowal (2014) Bouts dramatischen Lachens von Mrs. Bennet, dargestellt von der Schauspielerin Alison Steadman, in der BBC Mini-Fernsehserie Pride and Prejudice (Birtwistle und Langton 1995) analysiert. Das Korpus bestand aus mehr als einem Dutzend von Lachbeispielen, mit deren Hilfe Alison Steadman Mrs. Bennet als eine exaltierte, hysterische Frau charakterisierte. Es zeigte sich, dass diese Bouts jeweils aus einer Folge einzelner Calls bestanden, die ihrerseits rhythmische, auditiv unterscheidbare Segmente enthielten. Bouts wurden unter Verwendung der PRAAT-Software nach ihrer Gesamtdauer, der Dauer der einzelnen Segmente, der minimalen und maximalen f0/Segment, der Form der f0-Kontur und der Sprachqualität analysiert; hörbares Atmen, das sich direkt an das Lachen anschloss, wurde nicht in die Analysen mit einbezogen.

Die phonetischen Feinanalysen der Bouts ergaben, dass Mrs. Bennets Lachen durch die Kombination einer Reihe von ungewöhnlichen Eigenschaften charakterisiert war. Zu diesen Eigenschaften zählten erweiterter Stimmtonumfang (expanded pitch range), lagyngales Pfeifen (laryngeal whistle), Oktavsprünge (octave jumps) und ingressive Phonation (ingressive phonation) im Verbund mit reduzierter supralaryngaler Artikulation vor allem von zentralen Vokalen und ohne kontrollierte Artikulation von Konsonanten. Pompino-Marschall u.a. (2014) haben Mrs. Bennets Lach-Bouts zusammenfassend als gewissermaßen unartikulierte Vokalisierungen mit laryngaler Dehnung beschrieben. Auf diskursiver Analyseebene erwies sich dieses hysterische Lachen als wenig ansteckend, da Mrs. Bennets Gesprächspartner nur gelegentlich in ihr Lachen einstimmten. Schließlich ist davon auszugehen, dass Alison Steadman als Schauspielerin in ihrer Darstellung von Hysterie ihr Lachen absichtlich gebrauchte. Diese Ergebnisse und Interpretationen sind unserer Meinung nach insgesamt schwer mit Provines (2004: 215) allgemeiner Definition des Lachens aus evolutionsbiologischer Perspektive als ,instinktiver, ansteckender, stereotypischer, unbewusst gesteuerter Vokalisierung spielerischer sozialer Interaktion" in Übereinstimmung zu bringen.

\section{Schlussbemerkungen}

Schon vor Anbruch des zwanzigsten Jahrhunderts waren sich einige wenige Psychologen, die sich mit dem vokalen Lachverhalten von Erwachsenen beschäftigten, der Komplexität dieses Phänomens bewusst und hatten den Mut, sich inm empirisch zu nähern. Sowohl diese frühe als auch die hier beschriebene gegenwärtige Forschung weist eine Fülle von Annahmen über Funktionen und Ursachen des Lachens sowie verschiedene Methoden auf, um diese Annahmen empirisch zu überprüfen. Insgesamt 
zeigen sich in diesen Studien einige übereinstimmende empirische Ergebnisse: Vokales Lachen ist offenbar ein wichtiges paralinguistisches Verhalten, um persönliche Einstellungen zu oder Perspektiven gegenüber einem Gesprächsthema indirekt auszudrücken; es beeinflusst dialogische Interaktionen und wird seinerseits von innen beeinflusst; und die Art, Häufigkeit, Dauer und Funktion des Lachens scheinen häufig vom sozialen Geschlecht der Beteiligten beeinflusst zu werden. Gleichzeitig ist zu beobachten, dass es in der Psychologie an einer kontinuierlich entwickelten Forschungstradition zu diesem Thema fehlt. Auch gegenwärtig gibt es nur wenige PsychologInnen, die sich mit diesem Thema befasst haben. Unserer Meinung nach ist dieser Mangel in nicht unwesentlichem Maß darauf zurückzuführen, dass das Lachen eng mit sprachlichen Interaktionen in Dialogen verbunden ist. Solche Interaktionen sind jedoch ein noch relativ unerforschtes Gebiet der Psychologie, das selbst in der Sozialpsychologie vermieden wird (z.B. Holtgraves 2014: 2). Es scheint, dass viele PsychologInnen nicht mit dem Problem umgehen können, das der Psychologe Theo Herrmann (2006: 427) als „Kommunikations-Prozess-Dilemma“ der Sprachpsychologie bezeichnet hat: Weil die grundlegende Analyseeinheit der Psychologie traditionellerweise das Individuum war und noch ist, die grundlegende Analyseeinheit verbaler Kommunikation aber zwangsläufig das Paar (oder eine größere Gruppe) ist, sind die psychologischen Modelle, die sich auf Individuen beziehen, nicht kompatibel mit Modellen, die sich auf Paare beziehen, die miteinander kommunizieren. Wir betrachten unsere eigenen Untersuchungen des vokalen Lachens von Erwachsenen in dialogischer Interaktion als einen bescheidenen Anfang der systematischen empirischen Beschäftigung mit diesem interessanten Thema. In diesem Sinne stimmen wir mit Hall und Allin (1897) darin überein, „dass es kaum ein vielversprechenderes Thema für die psychologische Forschung gibt. [...] Wir müssen uns von Spekulationen lösen und unsere Theorien auf eine sehr breite empirische Basis stellen“ (40f.).

\section{Anmerkungen}

$1 \quad$ Alle Übersetzungen der englischsprachigen Quellen wurden von beiden Autorlnnen vorgenommen.

\section{Literatur}

Adams, Robert M. und Barbara Kirkevold (1978), „Looking, Smiling, Laughing, and Moving in Restaurants: Sex and Age Differences“. Environmental Psychology and Nonverbal Behavior 3: 117-121.

Adelswärd, Viveka und Britt-Marie Öberg (1998), „,The Function of Laughter and Joking in Negotiation Activities“. Humor 11: 411-429.

Allin, Arthur (1903), „On Laughter“. Psychological Review 10: 306-315. 
Allport, Floyd H. (1924), Social Psychology. New York: Houghton Mifflin.

Apte, Mahadev L. (1985), Humor and Laughter: An Anthropological Approach. Ithaca: Cornell University Press.

Arendt, Hannah (1964), Eichmann in Jerusalem: A Report on the Banality of Evil. New York: Viking.

Armstrong, Martin (1928), Laughing: An Essay. New York: Harper.

Bachorowski, Jo-Anne, Moria J. Smoski und Michael J. Owren (2001), „The Acoustic Features of Human Laughter". Journal of the Acoustical Society of America 110: 1581-1597.

Bergson, Henri (1900/1914), Le Rire: Essai sur la Signification du Comique. Paris: Alcan. Englisch von C. Brereton und F. Rothwell: Laughter: An Essay on the Meaning of the Comic. New York: Macmillan.

Black, Donald W. (1984), „Laughter“. JAMA 252: 2995-2998.

Bliss, Sylvia H. (1915), „The Origin of Laughter“. The American Journal of Psychology 26: 236-246.

Bühler, Karl (1934/1982), Sprachtheorie: Die Darstellungsfunktion der Sprache. Stuttgart: Gustav Fischer.

Carpenter, Ransom (1922), „Laughter, a Glory in Sanity“. The American Journal of Psychology 33: 419-422.

Chafe, Wallace (2007), The Importance of Not Being Earnest: The Feeling Behind Laughter and Humor. Amsterdam: John Benjamins.

Chapman, Anthony J. (1973a), „Funniness of Jokes, Canned Laughter und Recall Performance". Sociometry 36: 569-578.

Chapman, Anthony J. (1973b), „Social Facilitation of Laughter in Children“. Journal of Experimental Social Psychology 9: 528-541.

Chapman, Anthony und Wendy A. Chapman (1974), „Responsiveness to Humor: Its Dependency upon a Companion's Humorous Smiling and Laughter". The Journal of Psychology 88: 245-252.

Clayman, Steven E. und John Heritage (2002), The News Interview: Journalists and Public Figures on the Air. Cambridge: Cambridge University Press.

Clinton, Bill (2004), My Life. New York: Alfred A. Knopf.

Clinton, Hillary R. (2003), Living History. New York: Simon \& Schuster.

Crystal, David (1997), The Cambridge Encyclopedia of Language (2nd ed.). Cambridge: Cambridge University Press.

Diserens, Charles M. (1926), „Recent Theories of Laughter“. Psychological Bulletin 23: 247-255.

Diserens, Charles M. und Mabel Bonifield (1930), „Humor and the Ludicrous“. Psychological Bulletin 27: 99-118.

Duncan, Starkey und Donald W. Fiske (1977), Face-to-Face Interaction: Research, Methods, and Theory. Hillsdale, NJ: Lawrence Erlbaum.

Durant, John und Jonathan Miller (Hrsg.) (1988), Laughing Matters: A Serious Look at Humour. Essex: Longman Scientific \& Technical.

Flores, Reena (2014), „The Comprehensive Supercut of Hillary Clinton Laughing Awkwardly with Reporters“. URL: http://www.nationaljournal.com/politics/the-comprehensive-supercut-of-hillary-clinton 
Fridlund, Alan J. (1991), „Sociality of Solitary Smiling: Potentiation by an Implicit Audience“. Journal of Personality and Social Psychology 60: 229-240.

Fridlund, Alan J. und Jennifer M. Loftis (1990), „Relations between Tickling and Humorous Laughter: Preliminary Support for the Darwin-Hecker Hypothesis“. Biological Psychology 30: 141-150.

Gaus, Günter (1964), Zur Person: Porträts in Frage und Antwort. München: Feder.

Glenn, Phillip J. (2003), Laughter in Interaction. Cambridge: Cambridge University Press.

Glenn, Phillip und Elizabeth Holt (Hrsg.) (2013), Studies of Laughter in Interaction. London: Bloomsbury.

Goffman, Erving (1981), Forms of Talk. Philadelphia: University of Philadelphia Press.

Graumann, Carl F. und Werner Kallmeyer (2002), „Perspective and Perspectivation in Discourse: An Introduction“. In: Carl F. Graumann und Werner Kallmeyer (Hrsg.), Perspective and Perspectivation in Discourse. Amsterdam: John Benjamins: 1-11.

Gregory, Joshua C. (1924), The Nature of Laughter. London: Kegan Paul, Trench, Trubner \& Co.

Greig, John Young T. (1923/1969), The Psychology of Laughter and Comedy. London: Allen \& Unwin.

Grotjahn, Martin (1957), Beyond Laughter. New York: McGraw-Hill.

Hall, G. Stanley und Arthur Allin (1897), „The Psychology of Tickeling, Laughing, and the Comic“. The American Journal of Psychology 9: 1-44.

Harris, Christine R. und Nicholas Christenfeld (1997), „Humour, Tickle, and the DawinHecker Hypothesis“. Cognition and Emotion 11: 103-110.

Hayworth, Donald (1928), „The Social Origin and Function of Laughter“. Psychological Review 35: 367-384.

Hecker, Ewald (1873), Die Physiologie und Psychologie des Lachens und des Komischen: Ein Beitrag zur experimentellen Psychologie für Naturforscher, Philosophen und gebildete Laien. Berlin: Ferdinand Dümmler.

Herrmann, Theo (2006), „Dilemmata der Sprachpsychologie“. In: Kristel Proost und Edeltraud Winkler (Hrsg.), Von Intentionalität zur Bedeutung konventionalisierter Zeichen. Tübingen: Gunter Narr: 419-436.

Hertzler, Joyce O. (1970), Laughter: A Socio-Scientific Analysis. New York: Exposition. Holtgraves, Thomas M. (2014), „Language and Social Psychology: Introduction and Overview“. In: Thomas M. Holtgraves (Hrsg.), The Oxford Handbook of Language and Social Psychology. New York: Oxford University Press: 1-7.

Hopper, Robert (1992), Telephone Conversation. Bloomington, IN: Indiana University Press.

Jaffe, Eric (2010), „The Psychological Study of Smiling“. Association for Psychological Science 23: 12-18.

Jefferson, Gail (1979), „A Technique for Inviting Laughter and its Subsequent Acceptance/Declination“. In: George Psathas (Hrsg.), Everyday Language: Studies in Ethnomethodology. New York: Irvington: 79-96.

Jefferson, Gail (1984), „On the Organization of Laughter in Talk about Troubles“. In: J. Maxwell Atkinson und John Heritage (Hrsg.), Structures of Social Action: Studies in Conversation Analysis. Cambridge: Cambridge University Press: 346-369.

Jefferson, Gail, Harvey Sacks und Emmanuel Schegloff (1984), „On Laughter in Pursuit of Intimacy“. Working Paper Università di Urbino, no. 135, serie C. 
Kimmins, Charles W. (1928), The Springs of Laughter. London: Methuen.

Kotthoff, Helga (1996), „Vom Lächeln der Mona Lisa zum Lachen der Hyänen“. In: Helga Kotthoff (Hrsg.), Das Gelächter der Geschlechter: Humor und Macht in Gesprächen von Frauen und Männern. 2. Auflage. Konstanz: Universitätsverlag Konstanz: 121163.

Martin, Rod A. (2001), „Humor, Laughter, and Physical Health: Methodological Issues and Research Findings“. Psychological Bulletin 127: 504-519.

Martin, Rod A. (2006), The Psychology of Humor: An Integrative Approach. San Diego, CA: Elsevier.

Martin, G. Neil und Colin D. Gray (1996), „The Effects of Audience Laughter on Men's and Women's Responses to Humor". The Journal of Social Psychology 136: 221231.

Martin du Gard, Roger (1928/2003), Die Thibaults. Wien: Paul Zsolnay.

Merriam-Webster's Collegiate Dictionary. 11. Auflage. Springfield, MA: Merriam-Webster 2003.

Milner, George B. (1972), „Homo Ridens: Towards a Semiotic Theory of Humour and Laughter". Semiotica 5: 1-30.

Morrison, Jack (1940), „A Note Concerning Investigations on the Constancy of Audience Laughter". Sociometry 3: 179-185.

Nwokah, Evangeline E., Hui-chin Hsu, Patricia Davies und Alan Fogel (1999), „The Integration of Laughter and Speech in Vocal Communication: A Dynamic Systems Perspective“. Journal of Speech, Language, and Hearing Research 42: 880-894.

O'Connell, Daniel C. und Sabine Kowal (1998), „Orality and Literacy in Public Discourse: An Interview of Hannah Arendt“. Journal of Pragmatics 30: 543-564.

O'Connell, Daniel C. und Sabine Kowal (2004), „Hillary Clinton's Laughter in Media Interviews". Pragmatics 14: 463-478.

O’Connell, Daniel C. und Sabine Kowal (2005), „Laughter in Bill Clinton's My Life (2004) Interviews“. Pragmatics 15: 275-299.

O'Connell, Daniel C. und Sabine Kowal (2006), „Laughter in the Film The Third Man“. Pragmatics 16: 305-327.

O'Connell, Daniel C. und Sabine Kowal (2009), „Transcription Systems for Spoken Discourse“. In: Sigurd D'hondt, Jan-Ola Östman und Jef Verschueren (Hrsg.), The Pragmatics of Interaction. Amsterdam: John Benjamins.

O'Connell, Daniel C. und Sabine Kowal (2010), „Interjections in the Performance of Jane Austen's Pride and Prejudice“. Journal of Psycholinguistic Research 39: 285-304.

Panksepp, Jaak (2005), „Beyond a Joke: From Animal Laughter to Human Joy?" Science 308: 62-63.

Panksepp, Jaak (2007), „Neuroevolutionary Sources of Laughter and Social Joy: Modeling Primal Human Laughter in Laboratory Rats“. Behavioural Brain Research 182: 231-244.

Panksepp, Jaak und Jeff Burgdorf (2003), „Laughing Rats and the Evolutionary Antecedents of Human Joy?" Physiology \& Behavior 79: 533-547.

Partington, Alan (2006), The Linguistics of Laughter: A Corpus-Assisted Study of Laughter Talk. London: Routledge.

Piddington, Ralf (1933), The Psychology of Laughter: A Study of Social Adaptation. London: Figurehead. 
Pompino-Marschall, Bernd, Daniel C. O'Connell und Sabine Kowal (2014), „Prosody in Some Expressions of Emotion: Notes on the Phonetics of Laughter". In: Jürgen Trouvain und Nick Campbell (Hrsg.), Phonetics of Laughing. Saarbrücken: Saarland University Press.

Provine, Robert R. (1993), „Laughter Punctuates Speech: Linguistic, Social and Gender Contexts of Laughter". Ethology 95: 291-298.

Provine, Robert R. (2000), Laughter: A Scientific Investigation. New York: Penguin.

Provine, Robert R. (2000/2001), „The Laughing Species“. Natural History 109: 72-77.

Provine, Robert R. (2004), „Laughing, Tickling, and the Evolution of Speech and Self“. Current Directions in Psychological Science 13: 215-218.

Provine, Robert R. (2012), Curious Behavior: Yawning, Laughing, Hicupping, and Beyond. Cambridge, MA: The Belknap Press of Harvard University Press. Deutsch von S. Vogel: Ein seltsames Wesen: Warum wir gähnen, rülpsen, niesen und andere komische Dinge tun. Reinbek bei Hamburg: Rowohlt 2014.

Rhodes, Richard R. (1992), „Interjections“. In: William Bright (Hrsg.), International Encyclopedia of Linguistics. New York: Oxford University Press: 222.

Romaniuk, Tanya (2009), „The ,Clinton Cackle': Hillary Rodham Clinton's Laughter in News Interviews“. Crossroads of Language, Interaction, and Culture 7: 17-49.

Romaniuk, Tanya (2013), „Interviewee Laughter and Disaffiliation in Broadcast News Interviews“. In: Phillip Glenn und Elizabeth Holt (Hrsg.), Studies of Laughter in Interaction. London: Bloomsbury: 201-220.

Ruch,Willibald und Paul Ekman (2001), „The Expressive Pattern of Laughter“. In: Alfred Kaszniak (Hrsg.), Emotions, Qualia, and Consciousness. Singapore: World Scientific: 426-443.

Seibt, Gustav (2002), „Der Einspruch des Körpers: Philosophien des Lachens von Platon bis Plessner - und zurück“. Merkur 56: 751-762.

Sidis, Boris (1913), The Psychology of Laughter. New York: D. Appleton and Co.

Smoski, Moria J. und Jo-Anne Bachorowski (2003), „Antiphonal Laughter between Friends and Strangers“. Cognition and Emotion 17: 327-340.

Sully, James (1902/2012), An Essay on Laughter: Its Forms, its Causes, its Development and its Value. URL: www.forgottenbooks.org

Trouvain, Jürgen (2001), „Phonetics of 'Speech-Laughs'“. Proceedings of the Conference on Orality \& Gestuality (ORAGE), Aix-en-Provence (France): 634-639.

Trouvain, Jürgen (2003), „Segmenting Phonetic Units in Laughter“. 15 ${ }^{\text {th }}$ ICPhS Barcelona: 2793-2796.

Trouvain, Jürgen (2014), „Laughing, Breathing, Clicking - The Prosody of Nonverbal Vocalisations". Proceedings of Speech Prosody (SP7), Dublin.

Trouvain, Jürgen und Khiet P. Truong (2012), „Comparing Non-Verbal Vocalisations in Conversational Speech Corpora“. Proceedings of the $4^{\text {th }}$ International Workshop on Corpora for Research on Emotion Sentiment \& Social Signals, Istanbul: 36-39.

Vettin, Julia und Dietmar Todt (2004), „Laughter in Conversation: Features of Occurrence and Acoustic Structure“. Journal of Nonverbal Behavior 28: 93-115.

Washburn, R. W. (1929), „A Study of the Smiling and Laughing of Infants in the First Year of Life“. Genetic Psychology Monographs 6: 397-535.

Wertheimer, Michael (1979), A Brief History of Psychology (rev. ed.). New York: Holt, Rinehart and Winston. 
Wundt, Wilhelm (1862), Beiträge zur Theorie der Sinneswahrnehmungen. Leipzig:Winter.

\section{Filmographie}

Pride and Prejudice (UK: BBC 1995, Regie: Simon Langton, Produktion: Sue Birtwistle; DVD der TV-Serie).

The Third Man (UK 1949, Regie: Carol Reed, Produktion: Alexander Korda und David O. Selznik [Motion Picture]).

Der dritte Mann (D 1962, Regie: Carol Reed, Produktion: Alexander Korda und David O. Selznik [Deutsche Synchronisierung]).

Prof. Dr. Sabine Kowal

Roonstr. 29

D-12203 Berlin

E-Mail: kowal-berlin@t-online.de
Prof. Dr. Daniel C. O'Connell 3601 Lindell Blvd.

St. Louis, MO 63108, USA

E-Mail: doconnell@jesuits.org 